Original Research Paper

\title{
Inequalities Associated to a Sequence of Dyadic Martingales
}

\author{
Santosh Ghimire \\ Department of Applied Sciences and Chemical Engineering, Pulchowk Campus, Tribhuvan University, Nepal
}

\author{
Article history \\ Received: 03-09-2020 \\ Revised: 22-12-2020 \\ Accepted: $23-12-2020$ \\ Email: santoshghimire@ioe.edu.np
}

\begin{abstract}
In this article, we establish some inequalities associated to a sequence of dyadic martingales. These inequalities are sub-Gaussian type estimates. We derive the inequalities for a regular sequence of dyadic martingales and also for a tail sequence.
\end{abstract}

Keywords: Dyadic Martingales, Square Function, Tail Square Function

\section{Introduction}

We first discuss the meaning of the word 'martingale'. Originally martingale meant a strategy for betting in which you double your bet every time you lose. Let us consider a game in which the gambler wins his stake if a coin comes up heads and loses it if the coin comes up tails. The strategy is that the gambler doubles his bet every time he loses and continues the process, so that the first win would recover all previous losses plus win a profit equal to the original stake. This process of betting can be represented by a sequence of functions which is an example of dyadic martingale. Now we give the definition of dyadic martingales. For this let $\mathcal{D}_{n}$ denote the family of dyadic subintervals of the unit interval $[0,1)$ of the form $\left[\frac{j}{2^{n}}, \frac{j+1}{2^{n}}\right)$ where $n=0,1$, $2 \cdots$ and $j=0,1, \cdots 2^{n}-1$.

Definition 1.1 (Dyadic Martingale) (Bañuelos and Moore, 1999)

A dyadic martingale is a sequence of integrable functions, $\left\{f_{n}\right\}_{n=0}^{\infty}$ from $[0,1) \rightarrow \mathbb{R}$ such that:

(i) For every $n, f_{n}$ is $\mathfrak{F}_{n}$-measurable where $\mathfrak{F}_{n}$ is the $\sigma$ algebra generated by dyadic intervals of the form $\left[\frac{j}{2^{n}}, \frac{j+1}{2^{n}}\right), j \in\left\{0,1,2, \cdots 2^{n}-1\right\}$

(ii) And the following conditional expectation condition for all $n \geq 0$ holds:

$$
\mathbb{E}_{(}\left(f_{n+1} \mid \mathfrak{F}_{n}\right)=f_{n}
$$

$$
\begin{aligned}
& \text { where, } \quad \mathbb{E}\left(f_{n+1} \mid \mathfrak{F}_{n}\right)(x)=\frac{1}{\left|Q_{n}\right|} \int_{Q_{n}} f_{n+1}(y) d y, \quad \text { for } \\
& Q_{n} \in \mathcal{D}_{n} \text { and } x \in Q_{n} .
\end{aligned}
$$

A most general type of example of dyadic martingale is given by: Let $f \in L^{1}[0,1)$ and $Q_{n}$ be a dyadic interval of length $\frac{1}{2^{n}}$ on $[0,1)$. Define $f_{n}(x)=\frac{1}{\left|Q_{n}\right|} \int_{Q_{n}} f(y) d y, x \in Q_{n}$

where $\left|Q_{n}\right|$ is length of $Q_{n}$. Then $\left\{f_{n}\right\}_{n=1}^{\infty}$ is a dyadic martingale on $[0,1)$. We now prove that the functions so defined are a dyadic martingale.

For this, we note that $\mathfrak{F}_{0}=\{[0,1), \phi\}, \mathfrak{F}_{1}=\{[0,1)$, $\phi,[0,1 / 2),[1 / 2,1)\}$ and so on. We have $f_{n}(x)=$ $\frac{1}{\left|Q_{n}\right|} \int_{Q_{n}} f(y) d y, x \in Q_{n}$ and this is the average of $f$ on $Q_{n}$. Consequently, $f_{n}$ is constant on each of these $n$th generation dyadic intervals $Q_{n}=\left[\frac{j}{2^{n}}, \frac{j+1}{2^{n}}\right)$ where $n=0$, $1,2 \cdots$ and $j=0,1, \cdots 2^{n}-1$. Thus for all $\lambda \in \mathbb{R}$, the set $\left\{x \in[0,1): f_{n}(x)>\lambda\right\}$ belongs to $\mathfrak{F}_{n}$. Hence for each $n, f_{n}$ is $\mathfrak{F}_{n}$ measurable. This shows that the first condition is satisfied. Next, we show that the expectation condition is also satisfied. Here:

$$
\mathbb{E}\left(f_{n+1} \mid \mathfrak{F}_{n}\right)(x)=\frac{1}{\left|Q_{n}\right|} \int_{Q_{n}} f_{n+1}(y) d y
$$

where, $x \in Q_{n}$ and $\left|Q_{n}\right|=\frac{1}{2^{n}}$. Let $Q_{n+1,1}$ and $Q_{n+1,2}$ be the $(n+1)$ th generation dyadic intervals such that $Q_{n}=$ $Q_{n+1,1} \cup Q_{n+1,2}$. Using that fact that $f_{n+1}$ is constant on $Q_{n+1,1}$ and $Q_{n+1,2}$, we have: 


$$
\begin{aligned}
& \mathbb{E}\left(f_{n+1} \mid \mathfrak{F}_{n}\right)(x)=\frac{1}{\left|Q_{n}\right|} \int_{Q_{n+1,1} \cup Q_{n+1,2}} f_{n+1}(y) d y \\
& =\frac{1}{\left|Q_{n}\right|}\left[\int_{Q_{n+1,1}} f_{n+1}(y) d y+\int_{Q_{n+2,1}} f_{n+1}(y) d y\right] \\
& =\frac{1}{\left|Q_{n}\right|}\left[f_{n+1}(y)\left|Q_{n+1,1}\right|+f_{n+1}(y)\left|Q_{n+1,2}\right|\right] \\
& =\frac{1}{\left|Q_{n}\right|}\left[\left|Q_{n+1,1}\right| \frac{1}{\left|Q_{n+1,1}\right|} \int_{Q_{n+1,1}} f(y) d y+\left|Q_{n+1,2}\right| \frac{1}{\left|Q_{n+1,2}\right|} \int_{Q_{n+1,2}} f(y) d y\right] \\
& =\frac{1}{\left|Q_{n}\right|} \int_{Q_{n+1,1} \cup Q_{n+1,2}} f(y) d y \\
& =\frac{1}{\mid Q_{n}} \int_{Q_{n}} f(y) d y \\
& =f_{n}(x) .
\end{aligned}
$$

Hence the functions $f_{n}(x)=\frac{1}{\left|Q_{n}\right|} \int_{Q_{n}} f(y) d y, x \in Q_{n}$ is dyadic martingale.

Burkholder and Gundy (1970) proved $\{x: S f(x)<$ $\infty\} \stackrel{\text { a.e. }}{=}\left\{x: \lim f_{n}\right.$ exists $\}$ where $\stackrel{\text { a.e. }}{=}$ means the sets are equal upto a set of measure zero. From this result, we observe that dyadic martingales $\left\{f_{n}\right\}$ behave asymptotically well on the set $\{x: S f(x)<\infty\}$. But what can be said about the asymptotic behavior of dyadic martingales on the complement of this set? Its behavior is quite pathological on the set $\{x: S f(x)=\infty\}$. In particular it is unbounded a.e. on this set. In order to study the asymptotic behavior of the sequence of dyadic martingales, the martingales inequalities are helpful. These inequalities provide sub-Gaussian type estimates for the growth of the dyadic martingales. We derive these estimates for a regular sequence and a tail sequence of dyadic martingales. Asymptotic behavior of the martingales is studied through the law of the iterated logarithm of martingales (Stout, 1970). There is law of the iterated logarithm for various other contexts such as for harmonic functions, independent random variables, lacunary trigonometric series (Ghimire and Moore, 2014; Bañuelos et al., 1988). We now state our main results:

- Inequality 1 . For a dyadic martingale $\left\{f_{n}\right\}$ and $\lambda>0$ we have:

$$
\left|\left\{x \in[0,1): \sup _{m \geq 1}\left|f_{m}(x)\right|>\lambda\right\}\right| \leq 6 \exp \left(\frac{-\lambda^{2}}{2\|S f\|_{\infty}^{2}}\right) .
$$

- Inequality 2. For a dyadic martingale $\left\{f_{n}\right\}$, with $\lambda>$ 0 and, $n$ fixed positive integer we have:

$$
\left|\left\{x \in[0,1): \sup _{m \geq n}\left|f(x)-f_{m}(x)\right|>\lambda\right\}\right| \leq 12 \exp \left(\frac{-\lambda^{2}}{8\left\|S_{n}^{\prime} f\right\|_{\infty}^{2}}\right) .
$$

\section{Preliminaries}

We first fix some notations, give some definitions which will be used in the course of the proof.

\section{Definition 2.1}

For a dyadic martingale, $\left\{f_{n}\right\}_{n=0}^{\infty}$, we define:

(i) The increments: $d_{k}=f_{k}-f_{k-1}$, So $f_{n}(x)=\sum_{k=1}^{n} d_{k}(x)+$ $f_{0}(x)$

(ii) The quadratic characteristics or square function:

$$
S_{n}^{2} f(x)=\sum_{k=1}^{n} d_{k}^{2}(x)
$$

(iii) the limit function: $S^{2} f(x)=\lim _{n \rightarrow \infty} S_{n}^{2} f(x)=\sum_{k=1}^{n} d_{k}^{2}(x)$

(iv) the tail square function:

$$
S_{n}^{\prime 2} f(x)=\left(S_{n}^{\prime} f(x)\right)^{2}=\sum_{k=n+1}^{\infty} d_{k}^{2}(x) .
$$

The martingale square function is a local version of variance and can also be understood as a discrete counterpart of the area function in Harmonic Analysis. From the definition, we note that for any $x, y \in Q_{n}$, we have $S_{n}^{2} f(x)=S_{n}^{2} f(y)$. But the martingale tail square function, $S_{n}^{\prime 2} f(x)$ may not be equal to $S_{n}^{\prime 2} f(y)$. For more about martingales (Neveu and Speed, 1975).

\section{Definition 2.2 (Hardy-Littlewood Maximal Function)}

Let $f \in L^{p}\left(\mathbb{R}^{n}\right), 1 \leq p \leq \infty$. Then Hardy-Littlewood Maximal function associated to $f$, denoted by $M f$, is defined as:

$$
M f(x)=\sup _{r>0} \frac{1}{|B(x, r)|} \int_{B(x, r)}|f(y)| d y,
$$

where, $B(x, r)$ is the ball with center at $x$ and radius $r$.

\section{Proof of the Main Results}

We first prove a Lemma. This Lemma is also known as Rubin's Lemma (Pipher, 1993). The proof of this Lemma can also be found in (Chang et al., 1985). Here we give a proof of the Lemma using a different approach. Our proof is more analytic than the original probabilistic approach. We will use this Lemma in the proof of our inequalities. 


\section{Lemma 3}

For a dyadic martingale $\left\{f_{n}\right\}_{n=0}^{\infty}$, with $f_{0}=0$ :

$$
\int_{0}^{1} \exp \left(f_{n}(x)-\frac{1}{2} S_{n}^{2} f(x)\right) d x \leq 1
$$

\section{Proof of Lemma 3}

We claim that:

$$
g(n)=\int_{0}^{1} \exp \left(\sum_{k=0}^{n} d_{k}(x)-\frac{1}{2} \sum_{k=0}^{n} d_{k}^{2}(x)\right) d x
$$

is a decreasing function of $n$, Let $Q_{n j}$ be an arbitrary $n$th generation dyadic interval. We have $\sum_{k=0}^{n} d_{k}(x)=f_{n}(x)$ and $f_{n}$ is constant on $Q_{n j}$. Using this we have:

$$
\begin{aligned}
& g(n+1) \\
& =\sum_{j=0}^{2 n} \int_{Q_{i j}} \exp \left(\sum_{k=0}^{n+1} d_{k}(x)-\frac{1}{2} \sum_{k=0}^{n+1} d_{k}^{2}(x)\right) d x \\
& =\sum_{j=0}^{2 n} \int_{Q_{i j}} \exp \left(\sum_{k=0}^{n} d_{k}(x)-\frac{1}{2} \sum_{k=0}^{n} d_{k}^{2}(x)\right) \exp \left(d_{n+1}(x)-\frac{1}{2} d_{n+1}^{2}(x)\right) \\
& =\sum_{j=0}^{2 n}\left[\exp \left(\sum_{k=0}^{n} d_{k}(x)-\frac{1}{2} \sum_{k=0}^{n} d_{k}^{2}(x)\right)\right] \int_{Q_{i j}} \exp \left(d_{n+1}(x)-\frac{1}{2} d_{n+1}^{2}(x)\right) d x .
\end{aligned}
$$

Let $Q_{(n+1) j}^{\prime}$ and $Q_{(n+1) j}^{\prime \prime}$ be the dyadic subintervals of $Q_{n j}$. Suppose $d_{n+1}$ takes the value $\alpha$ on $Q_{(n+1) j}^{\prime}$. Then by the expectation condition, $d_{n+1}$ takes the value $-\alpha$ on $Q_{(n+1) j}^{\prime \prime}$. This gives:

$$
\begin{aligned}
& \int_{Q_{n j}} \exp \left(d_{n+1}(x)-\frac{1}{2} d_{n+1}^{2}(x)\right) d x \\
& =\int_{Q_{(n+1) j}^{\prime}} \exp \left(\alpha-\frac{1}{2} \alpha^{2}\right) d x+\int_{Q_{(n+1) j}^{n}} \exp \left(-\alpha-\frac{1}{2} \alpha^{2}\right) d x \\
& =\left[\exp \left(\alpha-\frac{1}{2} \alpha^{2}\right)+\exp \left(-\alpha-\frac{1}{2} \alpha^{2}\right)\right] \frac{1}{2^{n+1}} \\
& =2 \exp \left(-\frac{\alpha^{2}}{2}\right) \frac{e^{\alpha}+e^{-\alpha}}{2} \frac{1}{2^{n+1}} \\
& =2 \exp \left(-\frac{\alpha^{2}}{2}\right) \cosh \alpha \frac{1}{2^{n+1}} .
\end{aligned}
$$

Now using the elementary fact that $\cosh x \leq e^{\frac{x^{2}}{2}}$, we have:

$$
\begin{aligned}
& g(n+1) \leq \sum_{j=0}^{2^{n}}\left[\exp \left(\sum_{k=0}^{n} d_{k}(x)-\frac{1}{2} \sum_{k=0}^{n} d_{k}^{2}(x)\right)\right]_{Q_{\ell_{j}}} 2 \exp \left(-\frac{\alpha^{2}}{2}\right) \exp \left(\frac{\alpha^{2}}{2}\right) \frac{1}{2^{n+1}} \\
& =\sum_{j=0}^{2^{n}}\left[\exp \left(\sum_{k=0}^{n} d_{k}(x)-\frac{1}{2} \sum_{k=0}^{n} d_{k}^{2}(x)\right)\right]_{Q_{i j}}\left|Q_{n j}\right| \\
& =\sum_{j=0}^{2^{n}} \int_{Q_{i j}} \exp \left(\sum_{k=0}^{n} d_{k}(x)-\frac{1}{2} \sum_{k=0}^{n} d_{k}^{2}(x)\right) d x \\
& =g(n .)
\end{aligned}
$$

Let $Q_{11}$ and $Q_{12}$ be the dyadic subintervals of $Q_{0}$. Assume that $d_{1}$ takes value $\theta$ on $Q_{11}$ so that it takes value - $\theta$ on $Q_{12}$ :

$$
\begin{aligned}
& g(1)=\int_{0}^{1} \exp \left(d_{1}(x)-\frac{1}{2} d_{1}^{2}(x)\right) d x \\
& =\int_{0}^{\frac{1}{2}} \exp \left(\theta-\frac{1}{2} \theta^{2}\right) d x+\int_{\frac{1}{2}}^{1} \exp \left(-\theta-\frac{1}{2} \theta^{2}\right) d x \\
& =\exp \left(\theta-\frac{1}{2} \theta^{2}\right) \frac{1}{2}+\exp \left(-\theta-\frac{1}{2} \theta^{2}\right) \frac{1}{2} \\
& =\exp \left(-\frac{1}{2} \theta^{2}\right) \frac{\left(e^{\theta}+e^{-\theta}\right)}{2} \\
& =\exp \left(-\frac{1}{2} \theta^{2}\right) \cosh \theta \\
& \leq \exp \left(-\frac{1}{2} \theta^{2}\right) \exp \left(\frac{1}{2} \theta^{2}\right) \\
& =1 .
\end{aligned}
$$

Since $g(n)$ is decreasing and $g(1) \leq 1$ we conclude:

$$
\int_{0}^{1} \exp \left(\sum_{k=0}^{n} d_{k}(x)-\frac{1}{2} \sum_{k=0}^{n} d_{k}^{2}(x)\right) d x \leq 1 .
$$

Hence:

$$
\int_{0}^{1} \exp \left(f_{n}(x)-\frac{1}{2} S_{n}^{2} f(x)\right) d x \leq 1
$$

This completes the proof.

\section{Remark 4}

Note that if we rescale the sequence $\left\{f_{n}\right\}$ by $\lambda$, then Lemma 3 gives:

$$
\int_{0}^{1} \exp \left(\lambda f_{n}(x)-\frac{1}{2} \lambda^{2} S_{n}^{2} f(x)\right) d x \leq 1 .
$$

This shows that this lemma is an inhomogeneous type inequality. We won't need this fact in the sequel. 


\section{Proof of Inequality 1}

Fix $n$. Let $\lambda>0, \gamma>0$. Then for every $m \leq n$ :

$$
f_{m}(x)=\frac{1}{\left|Q_{m}\right|} \int_{Q_{m}} f_{n}(y) d y, x \in Q_{m},\left|Q_{m}\right|=\frac{1}{2^{m}} .
$$

Fix $x$ : Then $\sup _{1 \leq m \leq n}\left|f_{m}(x)\right| \leq M\left|f_{n}\right|(x)$ where $M f_{n}$ is the Hardy-Littlewood maximal function of $f_{n}$. Then using Jensen's inequality we have:

$$
\begin{aligned}
& \exp \left(\gamma\left|f_{m}(x)\right|\right)=\exp \left(\gamma\left|\int_{Q_{m}} f_{n}(y) d\left(\frac{y}{\left|Q_{m}\right|}\right)\right|\right) \\
& \leq \frac{1}{\left|Q_{m}\right|} \int_{Q_{m}} \exp \left(\gamma\left|f_{n}(y)\right|\right) d y \\
& \leq M\left(e^{\gamma\left|f_{m}(x)\right|}\right)(x) .
\end{aligned}
$$

Using the Hardy-Littlewood maximal estimate, we have:

$$
\begin{aligned}
& \left|\left\{x \in[0,1): \sup _{1 \leq m \leq n}\left|f_{m}(x)\right|>\lambda\right\}\right| \\
& =\left|\left\{x \in[0,1): \sup _{1 \leq m \leq n} e^{\gamma\left|f_{m}(x)\right|}>e^{\gamma \lambda}\right\}\right| \\
& \leq\left|\left\{x \in[0,1): M\left(e^{\gamma\left|f_{m}\right|}\right)(x)>e^{\gamma \lambda}\right\}\right| \\
& \leq \frac{3}{e^{\gamma \lambda}} \int_{0}^{1} \exp \left(\gamma\left|f_{n}(y)\right|\right) d y \\
& \leq \frac{3}{e^{\gamma \lambda}} \exp \left(\frac{\gamma^{2}}{2}\left\|S_{n} f\right\|_{\infty}^{2}\right) \int_{0}^{1} \exp \left(\gamma\left|f_{n}(y)\right|-\frac{\gamma^{2}}{2} S_{n}^{2} f(y)\right) d y .
\end{aligned}
$$

Using Lemma 3 we have:

$$
\begin{aligned}
& \int_{0}^{1} \exp \left(\gamma\left|f_{n}(y)\right|-\frac{\gamma^{2}}{2} S_{n}^{2} f\right) d y \\
& =\int_{\left\{y: f_{n}(y) \geq 0\right\}} \exp \left(\gamma f_{n}(y)-\frac{\gamma^{2}}{2} S_{n}^{2} f(y)\right) d y \\
& +\int_{\left\{y: f_{n}(y)<0\right\}} \exp \left(-\gamma f_{n}(y)-\frac{\gamma^{2}}{2} S_{n}^{2} f(y)\right) d y \\
& \leq \int_{0}^{1} \exp \left(\gamma f_{n}(y)-\frac{\gamma^{2}}{2} S_{n}^{2} f(y)\right) d y \\
& +\int_{0}^{1} \exp \left(-\gamma f_{n}(y)-\frac{\gamma^{2}}{2} S_{n}^{2} f(y)\right) d y \\
& \leq 2 .
\end{aligned}
$$

So:

$$
\left|\left\{x \in[0,1): \sup _{1 \leq m \leq n}\left|f_{m}(x)\right|>\lambda\right\}\right| \leq \frac{6}{e^{2 \lambda}} \exp \left(\frac{\gamma^{2}}{2}\left\|S_{n} f\right\|_{\infty}^{2}\right) .
$$

Choose $\gamma=\frac{\lambda}{\left\|S_{n} f\right\|_{\infty}^{2}}$. With this $\gamma$, the above inequality becomes:

$$
\left|\left\{x \in[0,1): \sup _{1 \leq m \leq n}\left|f_{m}(x)\right|>\lambda\right\}\right| \leq 6 \exp \left(\frac{-\lambda^{2}}{2\left\|S_{n} f\right\|_{\infty}^{2}}\right) .
$$

Note that for the dyadic martingale $\left\{f_{n}\right\}$ :

$$
S_{n}^{2} f(x)=\sum_{k=1}^{n} d_{k}^{2}(x) \rightarrow S^{2} f(x)=\sum_{k=1}^{\infty} d_{k}^{2}(x) .
$$

Consequently:

$$
\frac{-1}{2\left\|S_{n} f\right\|_{\infty}^{2}} \leq \frac{-1}{2\|S f\|_{\infty}^{2}} .
$$

Recall the continuity property of Lebesgue measure: If $\left\{E_{n}\right\}$ is a sequence of sets with $E_{n} \subset E_{n+1}$ for all $n$ and $E=\bigcup_{n=1}^{\infty} E_{n}$, then $|E|=\lim _{n \rightarrow \infty}\left|E_{n}\right|$. Using this we get:

$$
\left|\left\{x \in[0,1): \sup _{m \geq 1}\left|f_{m}(x)\right|>\lambda\right\}\right| \leq 6 \exp \left(\frac{-\lambda^{2}}{2\|S f\|_{\infty}^{2}}\right) .
$$

This completes the proof of the first inequality.

\section{Proof of Inequality 2}

Fix $n$. Define a sequence $\left\{g_{m}\right\}$ as follows:

$$
g_{m}(x)=\left\{\begin{array}{lr}
0, & \text { if } m \leq n \\
f_{m}(x)-f_{n}(x), & \text { if } m>n
\end{array}\right.
$$

We first show that $\left\{g_{m}\right\}$ is a dyadic martingale. Clearly for every $m, g_{m}$ is measurable with respect to the sigma algebra $\mathfrak{F}_{m}$ : Let $m>n$. Then using the fact that $f_{m}$ is constant on the cube $Q_{m}$ we have:

$$
\begin{aligned}
& E\left(g_{m+1} \mid \mathfrak{F}_{m}\right)(x)=\frac{1}{\left|Q_{m}\right|} \int_{Q_{m}}\left[f_{m+1}(x)-f_{n}(x)\right] d x \\
= & \frac{1}{\left|Q_{m}\right|} \int_{Q_{m}} f_{m+1}(x) d x-\frac{1}{\left|Q_{m}\right|} \int_{Q_{m}} f_{n}(x) d x \\
= & \frac{1}{\left|Q_{m}\right|} \int_{Q_{m}} f_{m+1}(x) d x-f_{n}(x) \\
= & f_{m}(x)-f_{n}(x) \\
= & g_{m}(x) .
\end{aligned}
$$


Thus we have $E\left(g_{m+1} \mid \mathfrak{F}_{m}\right)=g_{m}$. This shows that $\left\{g_{m}\right\}$ is a martingale. Then applying the inequality 1 for this martingale, we get:

$$
\left|\left\{x \in[0,1): \sup _{m \geq 1}\left|g_{m}(x)\right|>\lambda\right\}\right| \leq 6 \exp \left(\frac{-\lambda^{2}}{2\|S g\|_{\infty}^{2}}\right) .
$$

But, $g_{m}(x)=0$ for $m \leq n$. Hence:

$$
\left|\left\{x \in[0,1): \sup _{m \geq n}\left|g_{m}(x)\right|>\lambda\right\}\right| \leq 6 \exp \left(\frac{-\lambda^{2}}{2\|S g\|_{\infty}^{2}}\right) .
$$

Again:

$$
\begin{aligned}
& S^{2} g(x)=\sum_{k=0}^{\infty} d_{k}^{2}(x)=\sum_{k=0}^{\infty}\left[g_{k+1}(x)-g_{k}(x)\right]^{2} \\
& =\sum_{k=n}^{\infty}\left[g_{k+1}(x)-g_{k}(x)\right]^{2} \\
& =\sum_{k=n}^{\infty}\left[f_{k+1}(x)-f_{n}(x)-f_{k}(x)+f_{n}(x)\right]^{2} \\
& =\sum_{k=n+1}^{\infty}\left[f_{k+1}(x)-f_{k}(x)\right]^{2} \\
& =\sum_{k=n+1}^{\infty} d_{k}^{2}(x) \\
& =S_{n}^{\prime 2} f(x) .
\end{aligned}
$$

This gives:

$$
\left|\left\{x \in[0,1): \sup _{m \geq n}\left|g_{m}(x)\right|>\lambda\right\}\right| \leq 6 \exp \left(\frac{-\lambda^{2}}{2\left\|S_{n}^{\prime} f\right\|_{\infty}^{2}}\right) .
$$

i.e.:

$$
\left|\left\{x \in[0,1): \sup _{m \geq n}\left|f_{m}(x)-f_{n}(x)\right|>\lambda\right\}\right| \leq 6 \exp \left(\frac{-\lambda^{2}}{2\left\|S_{n}^{\prime} f\right\|_{\infty}^{2}}\right)
$$

Clearly:

$$
\left\{x:\left|f(x)-f_{n}(x)\right|>\lambda\right\} \subset\left\{x: \sup _{m \geq n}\left|f_{m}(x)-f_{n}(x)\right|>\lambda\right\}
$$

So we have:

$$
\left|\left\{x:\left|f(x)-f_{n}(x)\right|>\lambda\right\}\right| \leq\left|\left\{x: \sup _{m \geq n}\left|f_{m}(x)-f_{n}(x)\right|>\lambda\right\}\right| .
$$

Consequently: $\left|\left\{x:\left|f(x)-f_{n}(x)\right|>\lambda\right\}\right| \leq 6 \exp \left(\frac{-\lambda^{2}}{2\left\|S_{n}^{\prime} f\right\|_{\infty}^{2}}\right)$.

By the triangle inequality we have:

$$
\begin{aligned}
& \sup _{m \geq n}\left|f(x)-f_{m}(x)\right| \leq \sup _{m \geq n}\left(\left|f(x)-f_{n}(x)\right|+\left|f_{n}(x)-f_{m}(x)\right|\right) \\
& =\left|f(x)-f_{n}(x)\right|+\sup _{m \geq n}\left|f_{n}(x)-f_{m}(x)\right| .
\end{aligned}
$$

This gives:

$$
\begin{aligned}
& \left\{x: \sup _{m \geq n}\left|f(x)-f_{m}(x)\right|>\lambda\right\} \\
& \subset\left\{x: \sup _{m \geq n}\left|f(x)-f_{n}(x)\right|>\frac{\lambda}{2}\right\} \cup\left\{x: \sup _{m \geq n}\left|f_{n}(x)-f_{m}(x)\right|>\frac{\lambda}{2}\right\}
\end{aligned}
$$

Therefore:

$$
\begin{aligned}
& \left|\left\{x: \sup _{m \geq n}\left|f(x)-f_{m}(x)\right|>\lambda\right\}\right| \\
& \leq\left|\left\{x:\left|f(x)-f_{m}(x)\right|>\frac{\lambda}{2}\right\}\right|+\left|\left\{x: \sup _{m \geq n}\left|f_{n}(x)-f_{m}(x)\right|>\frac{\lambda}{2}\right\}\right| .
\end{aligned}
$$

Then using (2) and (3) in the above inequality we get:

$$
\begin{aligned}
& \left|\left\{x: \sup _{m \geq n}\left|f(x)-f_{m}(x)\right|>\lambda\right\}\right| \\
& \leq 6 \exp \left(\frac{-\left(\frac{\lambda}{2}\right)^{2}}{2\left\|S_{n}^{\prime} f\right\|_{\infty}^{2}}\right)+6 \exp \left(\frac{-\left(\frac{\lambda}{2}\right)^{2}}{2\left\|S_{n}^{\prime} f\right\|_{\infty}^{2}}\right) \\
& =12 \exp \left(\frac{-\lambda^{2}}{8\left\|S_{n}^{\prime} f\right\|_{\infty}^{2}}\right) .
\end{aligned}
$$

Thus:

$$
\left|\left\{x: \sup _{m \geq n}\left|f(x)-f_{m}(x)\right|>\lambda\right\}\right| \leq 12 \exp \left(\frac{-\lambda^{2}}{8\left\|S_{n}^{\prime} f\right\|_{\infty}^{2}}\right) .
$$

This completes the proof of inequality 2 .

\section{Acknowledgement}

The author would like to thank Prof. Charles N. Moore (Ph. D. thesis advisor) of Washington State University, USA for his valuable suggestions on this manuscript. In 
addition, author thanks the chief editor of the journal and all the anonymous reviewers for their useful comments and suggestions. Their comments and suggestions greatly helped to improve the manuscript.

\section{Ethics}

This is a mathematical research article. No ethical issues will arise after the publication of the article.

\section{References}

Bañuelos, R., Klemes, I., \& Moore, C. N. (1988). An analogue for harmonic functions of Kolmogorov's law of the iterated logarithm. Duke Mathematical Journal, 57(1), 37-68.

Bañuelos, R., \& Moore, C. N. (1999). Probabilistic behavior of harmonic functions (Vol. 175). Springer Science \& Business Media.

Burkholder, D. L., \& Gundy, R. F. (1970). Extrapolation and interpolation of quasi-linear operators on martingales. Acta mathematica, 124(1), 249-304.
Chang, S. Y. A., Wilson, J. M., \& Wolff, T. H. (1985). Some weighted norm inequalities concerning the Schrödinger operators. Commentarii Mathematici Helvetici, 60(1), 217-246.

Ghimire, S., \& Moore, C. (2014). A lower bound in the tail law of the iterated logarithm for lacunary trigonometric series. Proceedings of the American Mathematical Society, 142(9), 3207-3216.

Neveu, J., \& Speed, T. P. (1975). Discrete-parameter martingales (Vol. 10). Amsterdam: North-Holland.

Pipher, J. (1993). A martingale inequality related to exponential square integrability. Proceedings of the American Mathematical Society, 118(2), 541-546.

Stout, W. F. (1970). A martingale analogue of Kolmogorov's law of the iterated logarithm. Zeitschrift für Wahrscheinlichkeitstheorie und verwandte Gebiete, 15(4), 279-290. 